4th International Conference Photoinduced Phase Transitions and Cooperative Phenomena, Wrocław 2011

\title{
Novel Excitation-Density Dependence in Strongly Spin-Charge Coupled System
}

\author{
J. OHARA ${ }^{a, b}$, Y. KANAMORI ${ }^{a}$ AND S. IshihARA ${ }^{a, b}$ \\ ${ }^{a}$ Department of Physics, Tohoku University, Sendai 980-8578, Japan \\ ${ }^{b}$ Core Research for Evolutional Science and Technology (CREST), Sendai 980-8578, Japan
}

\begin{abstract}
Photoinduced spin-charge dynamics in strongly correlated electron systems is studied based on an extended double-exchange model. Solving a time-dependent Schrödinger equation with the Lanczos method, we trace the process of photoinduced melting of an antiferromagnetic charge order and analyze the excitation-density dependence on that. In the case of low density photoexcitation, both charge and spin orders are melted by photocarrier doping. This is interpreted with a conventional double-exchange mechanism. In the case of high density photoexcitation, however, the charge order is melted. The antiferromagnetic spin order is transiently weakened but after turnoff of the photoirradiation it recovers. This phenomenon strikingly differs from the weak photoexcitation case.
\end{abstract}

PACS: 71.30. $+\mathrm{h}, 71.10 .-\mathrm{w}, 78.47 . \mathrm{J}-, 78.20 . \mathrm{Bh}$

\section{Introduction}

Optical tuning of physical properties such as an electric conductivity, a magnetism, and an electric polarization, is one of the most challenging themes in modern physics. Many efforts to a new material synthesis, a development of measurement technique, and a progress of theoretical method, have been devoted to achievement of such purpose. Strongly correlated electron systems where the cooperation and/or the competition among several degrees of freedom (charge, spin, and orbital etc.) play a crucial role for several physical properties are the ideal stages for such researching. Quantum dynamics induced by photoirradiation has been vigorously investigated.

One of the widely known examples are perovskite-type manganese oxides $\mathrm{R}_{1-x} \mathrm{~A}_{x} \mathrm{MnO}_{3}[\mathrm{R}=\mathrm{La}, \mathrm{Pr}$, $\mathrm{Nd}$ (rare-earth ions); $\mathrm{A}=\mathrm{Ca}, \mathrm{Sr}$ (alkaline earth ions)]. The $3 d$ orbitals of $\mathrm{Mn}$ ions split into the $e_{\mathrm{g}}$ orbitals and the $t_{2 \mathrm{~g}}$ orbitals because of the crystalline field by surrounding oxygen ions. The $e_{\mathrm{g}}$ electrons move about in the crystal, whereas the $t_{2 \mathrm{~g}}$ electrons behave as localized spins. The electron configuration in $\mathrm{Mn}$ ion is expressed as $\left(e_{\mathrm{g}}\right)^{1-x}\left(t_{2 \mathrm{~g}}\right)^{3}$. Around $x=0.5$, the system exhibits a charge-ordered insulating state associated with a long-range antiferromagnetic order of $t_{2 \mathrm{~g}}$ spins, and is in the vicinity of the phase boundary with a metallic state accompanied by a long-range ferromagnetic order [1]. The former and latter states are called as the antiferromagnetic charge-ordered (AFCO) state and the ferromagnetic metallic (FM) state, respectively.

In such a situation where the two states highly compete with each other, the photoinduced phase transition from the AFCO state to the FM state has been observed in the optical reflectivity and the optical Kerr rotation measurements $[2,3]$. Since the $t_{2 \mathrm{~g}}$ localized spins are strongly coupled with the itinerant $e_{\mathrm{g}}$ electrons which interact with the photon directly, melting of the charge order is associated with change in magnetic structure. Recently, Ichikawa et al. [4] have succeeded in the observation of a new type photoinduced charge-orbital ordered state in $\mathrm{Nd}_{0.5} \mathrm{Sr}_{0.5} \mathrm{MnO}_{3}$ with a picosecond time-resolved X-ray diffraction measurement. This state transiently emerges only by photoexcitation, and is a hidden insulating state distinct from a thermally induced state.

Thus, the photoinduced dynamics in correlated electron systems coupled with localized spins is more interesting. In order to understand such phenomena theoretically, we have investigated the changes in electronic structures with photoinduced AFCO to FM phase transition and the formation mechanisms of ferromagnetic spin-ordered domains in previous works [5-7]. In this paper, treating an extended double-exchange model, we carry out the research for an excitation-density dependence on the photoinduced melting of charge and spin orders and find a novel photoirradiation effect on the AFCO state.

\section{Model and method}

Employing the extended double-exchange model and assuming strong Hund's coupling limit, we treat the strongly spin-charge coupled system described by the effective spinless Hamiltonian [8] where conduction electrons are treated as spinless fermions. This is given by

$$
\begin{aligned}
\mathcal{H} & =\sum_{i}\left[\left(t_{i, i+1} c_{i}^{\dagger} c_{i+1}+\text { H.c. }\right)+V n_{i} n_{i+1}\right. \\
& \left.+J \boldsymbol{S}_{i} \cdot \boldsymbol{S}_{i+1}\right]
\end{aligned}
$$

with 


$$
\begin{aligned}
& t_{i, i+1}=-t\left[\exp \left(\mathrm{i} \frac{\left(\phi_{i}-\phi_{i+1}\right)}{2}\right) \cos \frac{\theta_{i}}{2} \cos \frac{\theta_{i+1}}{2}\right. \\
& \left.+\exp \left(-\mathrm{i} \frac{\left(\phi_{i}-\phi_{i+1}\right)}{2}\right) \sin \frac{\theta_{i}}{2} \sin \frac{\theta_{i+1}}{2}\right],
\end{aligned}
$$

where $c_{i}^{\dagger}$ creates a spinless fermion at the site $i$ and a fermion number operator is defined as $n_{i}=c_{i}^{\dagger} c_{i}$. A localized spin at the site $i$ is denoted as $\boldsymbol{S}_{i}$ with an amplitude of $S=1 / 2$, and $\left\{\theta_{i}, \phi_{i}\right\}$ are the angles of the spin in a polar representation. The itinerant electrons and the localized spins are regarded as the $e_{\mathrm{g}}$ electrons and the $t_{2 \mathrm{~g}}$ spins, respectively. We introduce the transfer integral $t_{i, i+1}$, the intersite Coulomb repulsion $V$, and the antiferromagnetic superexchange interaction $J$. The magnitude of transfer integral between the site $i$ and site $i+1$ is given by

$$
\left|t_{i, i+1}\right|=t \cos \frac{\theta_{i, i+1}}{2}
$$

where $\theta_{i, i+1}$ is the relative angle between $\boldsymbol{S}_{i}$ and $\boldsymbol{S}_{i+1}$. Since the value of $\left|t_{i, i+1}\right| / t$ takes its maximum (minimum) at $\theta_{i, i+1}=0(\pi)$, the ferromagnetic (antiferromagnetic) spin order favors the metallic (insulating) states in the conduction electron system. Thus the double-exchange interaction provided by the cooperation of the Hund coupling and the electron hopping, is expressed by the above relation. In the numerical calculations, the energy and time are scaled by $t$ and $t^{-1}$, respectively. We set $V / t=1.40$ and $J / t=1.24$ to reproduce the AFCO ground state, and the spin angles are determined so as to minimize the ground-state energy.

Interaction between photon and electron is introduced in the Peierls phase in the transfer term as $t_{i, i+1} \rightarrow$ $t_{i, i+1} \mathrm{e}^{-\mathrm{i} \int A(\tau) \mathrm{d} r}$. The vector potential at time $\tau$ is assumed to be a damped oscillator form as $A(\tau)=$ $A_{0} \mathrm{e}^{-\gamma_{0}^{2}\left(\tau-\tau_{0}\right)^{2}} \cos \omega_{0}\left(\tau-\tau_{0}\right)$ where $\gamma_{0}$ and $\tau_{0}$ are respectively a damping factor and a center of the wave packet. An intensity of the light is tuned by $A_{0}$, and $\omega_{0}$ is the photon energy. The time evolution of the electronic wave function is obtained by solving the time-dependent Schrödinger equation given by

$$
\mathrm{i} \frac{\mathrm{d}|\Psi(\tau)\rangle}{\mathrm{d} \tau}=\mathcal{H}|\Psi(\tau)\rangle
$$

Here we employ the exact-diagonalization method in finite size cluster based on the Lanczos method [9]. The time evolution of the localized spins is calculated by the classical equation of motion as follows:

$$
\begin{gathered}
\frac{\mathrm{d}}{\mathrm{d} \tau} \frac{\partial \mathcal{L}}{\partial \phi_{i}}-\frac{\partial \mathcal{L}}{\partial \dot{\phi}_{i}}=0, \\
\frac{\mathrm{d}}{\mathrm{d} \tau} \frac{\partial \mathcal{L}}{\partial \theta_{i}}-\frac{\partial \mathcal{L}}{\partial \dot{\theta}_{i}}=0,
\end{gathered}
$$

with

$$
\mathcal{L}=S \sum_{i} \cos \theta_{i} \dot{\phi}_{i}-\langle\mathcal{H}\rangle
$$

Here $\mathcal{L}$ denotes a Lagrangian of the system [10] and $\langle\ldots\rangle$ means the expectation value with respect to $|\Psi(\tau)\rangle$. Equations (2.5) and (2.6) are numerically solved within the fourth order Runge-Kutta method. For simplicity, we adopt the one-dimensional cluster. We have checked that the qualitatively similar results are obtained in the two-dimensional clusters. A system size in the cluster is taken to be $N=15$ and a number of conduction electrons is $N_{\text {ele }}=(N+1) / 2$ which corresponds to a half filling in the open-boundary condition. The pumping energy is tuned at $\omega_{0}=1.1 t$ with $\tau_{0}=50 t^{-1}$ and $\gamma_{0}=0.1 t$, which is equivalent to the main-peak energy of the optical absorption spectrum.

\section{Result and discussion}

In Fig. 1, we show the excitation-density dependence on the time evolution of the charge and spin structures. An absorbed-photon density $n_{\mathrm{ph}}$, a charge correlation function $K_{N}$, and a spin correlation function $K_{S}$ are defined as

$$
\begin{aligned}
n_{\mathrm{ph}}(\tau) & =\frac{\langle\mathcal{H}\rangle-E^{\mathrm{G} . \mathrm{S} .}}{N \omega_{0}}, \\
K_{N}(\tau) & =\frac{1}{N_{\text {bond }}} \sum_{i}\left\langle n_{i} n_{i+1}\right\rangle, \\
K_{S}(\tau) & =\frac{1}{N_{\text {bond }} S^{2}} \sum_{i} \boldsymbol{S}_{i} \cdot \boldsymbol{S}_{i+1},
\end{aligned}
$$

respectively. Here $E^{\text {G.S. }}$ and $N_{\text {bond }}$ denote the AFCO ground-state energy and a total number of the nearest-neighbor bonds, respectively. The absorbed-photon density monotonically increases with the light intensity $A_{0}$ as shown in Fig. 1a. As shown in Fig. 1b, the charge correlation functions increase by the photoirradiation. The charge-order melts monotonically by increasing the number of the doped photocarriers. Meanwhile the photon-density dependence on the spin dynamics strikingly differs from that on the charge dynamics (Fig. 1c). In the case of low density photoexcitation, $A_{0}=0.04$ and 0.08 , the $\mathrm{AF}$ spin orders are collapsed after photoirradiation as well as the charge orders. In the case of high density photoexcitation, $A_{0}=0.25$ and 0.35 , however, the AF spin orders are transiently weakened but after turnoff of the photoirradiation they recover. Unlike the charge order, the AF spin order tends to be hardened against the light intensity. We also carried out the same analyses for $N=15(=5 \times 3)$ ladder and $N=25$ chain systems, and confirmed qualitatively the same behaviors as the present system. The above features do not depend on the lattice-geometry and the cluster size.

We plot spatial charge and spin configurations before and after pumping in Fig. $1 \mathrm{~d}-\mathrm{f}$. The snapshots at $\tau t=85$ (immediately after turnoff of the photoirradiation) with $A_{0}=0.08$ (Fig. 1e) and $A_{0}=0.35$ (Fig. 1f) are shown as the weak photoexcitation case and the strong photoexcitation case, respectively. In Fig. 1e, the AF spin correlations are partially weakened in comparison with those at $\tau t=0$ (Fig. 1d), and an amplitude of the double-periodic charge density wave is also suppressed. The 


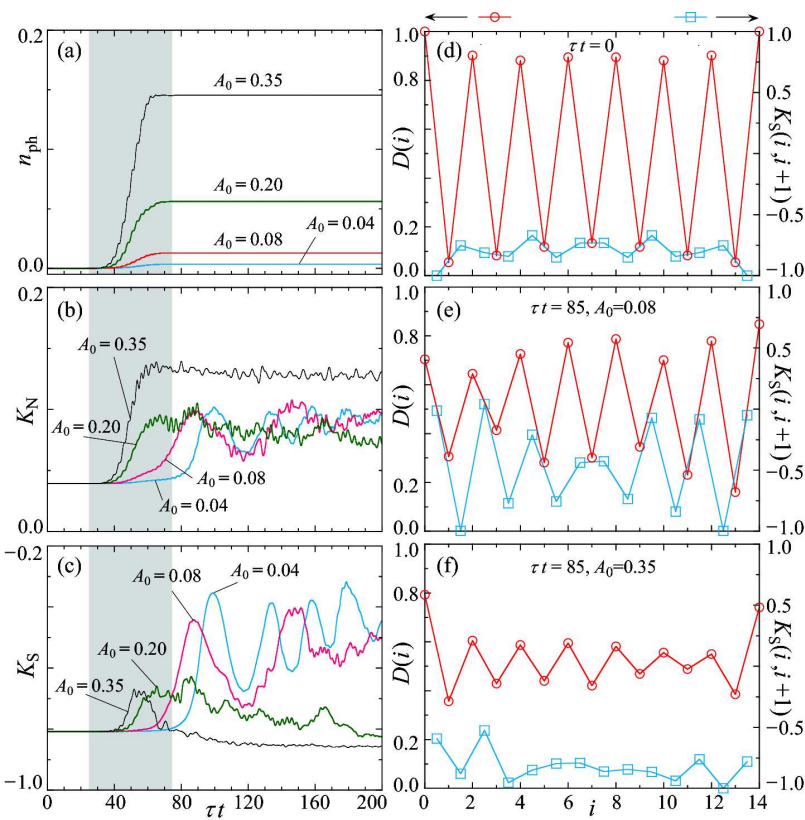

Fig. 1. Time variations of (a) absorbed-photon densities, (b) charge correlation functions, and (c) spin correlation functions under photoirradiation. The shaded regions indicate the period of photoirradiation. Spatial charge and spin configurations at (d) $\tau t=0$, (e) $\tau t=85$ with $A_{0}=0.08$, and (f) $\tau t=85$ with $A_{0}=0.35$. Charge density on $i$-th site and spin correlation between $i$ - and $i+1$-th sites are defined as $D(i)=\left\langle n_{i}\right\rangle$ and $K_{S}(i, i+1)=\boldsymbol{S}_{i} \cdot \boldsymbol{S}_{i+1} / S^{2}$, respectively.

observed changes in the spin structure are interpreted as the photogeneration of the ferromagnetic domains. This state corresponds to the island state which is adjacent to the AFCO state in a ground-state phase diagram [6]. After strong photoexcitation, entirely-different spatial structures are seen in Fig. 1f. The spin structure almost keeps the initial AF spin order (rather the AF spin order is slightly strengthened) despite the complete melting of charge order.

In Fig. 2, we show the optical absorption spectra before and after pumping. These are defined as

$$
\sigma(\omega)=-\frac{1}{N \pi} \operatorname{Im}\left\langle\mathcal{J} \frac{1}{\omega-\mathcal{H}+\langle\mathcal{H}\rangle+\mathrm{i} \eta} \mathcal{J}\right\rangle,
$$

with the current operator $\mathcal{J}=\mathrm{i} \sum_{i}\left(t_{i, i+1} c_{i}^{\dagger} c_{i+1}-\right.$ c.c. $)$. At $\tau t=0$, the spectrum has the insulating gap originating from the ground-state charge order. After weak photoexcitation, the low-energy oscillator strength grows up as shown in Fig. 2a. This increase of the spectra implies that the system becomes more conductive. In Fig. 2b, the spectral weight entirely decreases after strong photoexcitation. It reflects the fact that the strong AF spin order encumbers the motion of conducting electrons as represented by Eq. (2.3). Thus, the system does not exhibit a metallic behavior even in the charge-disordered state. This state is identified as an antiferromagnetic charge-disordered (AFCD) state.

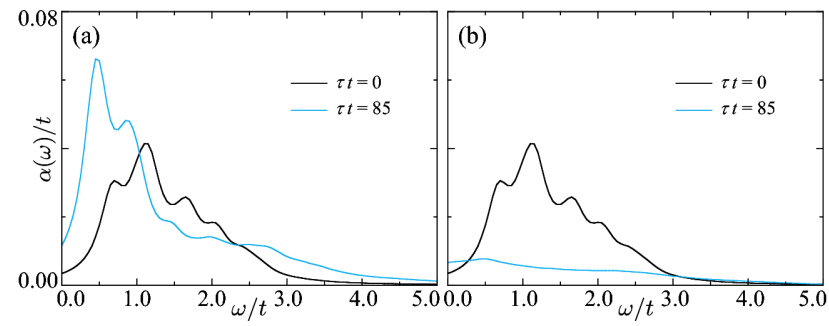

Fig. 2. Optical absorption spectra before $(\tau t=0)$ and after $(\tau t=85)$ pumping with $\eta=0.1 t$. The intensities of the pump light are chosen to be (a) $A_{0}=0.08$ and (b) $A_{0}=0.35$.

The microscopic electronic structures after weak and strong photoexcitation are summarized. In the former case, both charge and spin orders are melted. This melting demonstrates the photoinduced AFCO to FM transition. It is interpreted that the motion of photodoped carriers brings about the magnetic structure change through the double-exchange interaction. In the latter case, only charge order is melted, and the initial AFCO state changes into the AFCD state that never emerges as a static state. This fact indicates the existence of a hidden state which can be formed only by the high photodoping. We further revealed that the distributions of photodoped carriers in the band are qualitatively different between the weak and strong photoexcitation cases. In the weak photoexcitation, the holes are photodoped around top of the electron band, that is, the electrons in highest occupied levels are pumped up by the photoirradiation. On the other hand, in the strong photoexcitation, the electrons around bottom of the band are pumped up. In such the highly photoexcited state, the band narrowing is expected, in highly contrast to the weak photoexcitation case. It brings down the kinetic energy in the electron system and stabilizes the AF spin order. This is a possible scenario of the emergence of the AFCD state by the strong photoexcitation. Further calculations, such as the real-time tracking of the band structures and so on, are required in future work to elucidate the mechanism of these photoinduced dynamics completely.

\section{Summary}

We have investigated the photoinduced spin-charge dynamics in strongly correlated electron systems described by the double-exchange model. The photoirradiation effects on the AFCO state depend strongly on the photon density, that is, the weak photoexcitation triggers the transition into the FM state, whereas the strong photoexcitation induces the transition into the AFCD state. Especially the latter case indicates that the hidden state may appear by the high-density photoexcitation. We suggest that the "excitation density" is a key to search for a hidden state. 


\section{Acknowledgments}

The authors are grateful to H. Matsueda, S. Iwai, K. Yonemitsu, Y. Okimoto, and S. Koshihara for fruitful discussions. This research is supported by JSPS KAKENHI, DYCE (Optical Science of Dynamically Correlated Electrons) from MEXT, Next Generation Supercomputer Project, and CREST. Part of the numerical calculations was performed in the supercomputing facilities in ISSP, University of Tokyo and Kyoto University.

\section{References}

[1] M. Imada, A. Fujimori, Y. Tokura, Rev. Mod. Phys. 70, 1039 (1998).

[2] M. Fiebig, K. Miyano, Y. Tomioka, Y. Tokura, Science 280, 1925 (1998).

[3] M. Matsubara, Y. Okimoto, T. Ogasawara, Y. Tomioka, H. Okamoto, Y. Tokura, Phys. Rev. Lett. 99, 207401 (2007).
[4] H. Ichikawa, S. Nozawa, T. Sato, A. Tomita, K. Ichiyanagi, M. Chollet, L. Guerin, N. Dean, A. Cavalleri, S. Adachi, T. Arima, H. Sawa, Y. Ogimoto, M. Nakamura, R. Tamaki, K. Miyano, S. Koshihara, Nature Mater. 10, 101 (2011).

[5] Y. Kanamori, H. Matsueda, S. Ishihara, Phys. Rev. Lett. 103, 267401 (2009).

[6] Y. Kanamori, H. Matsueda, S. Ishihara, Phys. Rev. B 82, 115101 (2010).

[7] K. Satoh, S. Ishihara, J. Magn. Magn. Mater. 310, 798 (2007).

[8] P.W. Anderson, H. Hasegawa, Phys. Rev. 100, 675 (1955).

[9] T.J. Park, J.C. Light, J. Chem. Phys. 85, 5870 (1986).

[10] K. Obata, G. Tatara, Phys. Rev. B 77, 214429 (2008). 